\title{
Developmentally adapted cognitive processing therapy for adolescents and young adults with PTSD symptoms after physical and sexual abuse: study protocol for a randomized controlled trial
}

\author{
Rita Rosner ${ }^{1 *}$, Hans-Helmut König ${ }^{2}$, Frank Neuner ${ }^{3}$, Ulrike Schmidt ${ }^{4}$ and Regina Steil ${ }^{5}$
}

\begin{abstract}
Background: Although childhood sexual and/or physical abuse (CSA/CPA) is known to have severe psychopathological consequences, there is little evidence on psychotherapeutic interventions for adolescents and young adults suffering from post-traumatic stress disorder (PTSD). Equally sparse are data on moderators of treatment response on PTSD-related epigenetic changes, health care costs and loss of productivity, alterations in cognitive processing, and on how successful interventions affect all of these factors. Early treatment may prevent later (co)morbidity. In this paper, we present a study protocol for the evaluation of a newly developed psychotherapeutic manual for PTSD after CSA/CPA in adolescents and young adults - the Developmentally Adapted Cognitive Processing Therapy (D-CPT).
\end{abstract}

Methods/design: In a multicenter randomized controlled trial (RCT) D-CPT is compared to treatment as usual (TAU). A sample of 90 adolescent outpatients aged 14 to 21 years will be randomized to one of these conditions. Four assessments will be carried out at baseline, at end of treatment, and 3 and 6 months after end of therapy. Each time, patients will be assessed via clinical interviews and a wide range of questionnaires. In addition to PTSD symptoms and comorbidities, we will evaluate moderators of treatment response, epigenetic profiles, direct and indirect costs of this disorder, and neurophysiological processing of threat cues in PTSD and their respective changes in the course of these two treatments (D-CPT and TAU).

Discussion: The study will provide new insights in the understudied field of PTSD in adolescents and young adults. A newly developed intervention will be evaluated in this therapeutically underserved population. Results will provide data on treatment efficacy, direct and indirect treatment costs, as well as on associations of treatment outcome and PTSD intensity both to epigenetic profiles and to the neurobiological processing of threat cues. Besides, they will help to learn more about the psychopathology and possible new objective correlates of PTSD.

Trial registration: Germanctr.de identifier: DRKS00004787.

Keywords: Abuse, Adolescents, Cognitive processing therapy, Post-traumatic stress disorder

\section{Background}

Unfortunately, traumatic experiences in childhood and adolescence are common: a meta-analysis based on 65 international studies indicates that nearly $20 \%$ of women and $8 \%$ of men experienced sexual abuse prior to the age of 18 [1]. Regarding physical abuse in Western and

\footnotetext{
* Correspondence: rita.rosner@ku.de

'Department of Clinical and Biological Psychology, Catholic University

Eichstätt-Ingolstadt, Ostenstr. 25, Eichstätt 85071, Germany

Full list of author information is available at the end of the article
}

European nations, numbers range from 3.6\% to $16.3 \%$ [2-5]. These negative childhood experiences do not only have short-term consequences concerning the victims' mental health - they are also related to a number of psychiatric disorders during the whole life span [6]. Consequently, people exposed to childhood sexual abuse (CSA) have a 2.4 heightened risk for the development of psychopathology compared to those without such experiences. For childhood physical abuse (CPA), this factor is around 1.5 [7]. In a meta-analysis, mortality and morbidity, drug 
and alcohol misuse, risky sexual behavior, obesity, and criminal behavior were found as consequences of childhood maltreatment [8]. According to Cutajar et al. [9], CSA increases the risk for several mental diseases like psychosis, affective, anxiety, substance abuse, and personality disorders. There is a particularly high probability for the development of post-traumatic stress disorders (PTSD) exposure to CSA leads to an increased PTSD risk of 5.6 compared to non-CSA exposure. Accordingly, international studies show a high prevalence for PTSD after CSA, which ranges from $37 \%$ to $44 \%[10,11]$. However, victims of CSA do not only suffer from PTSD; comorbidity secondary to PTSD often develops during adolescence and early adulthood, moderated by the patient's efforts to fight painful trauma-related emotions, for instance by using harmful substances and by developing suicidal, self-injurious, or other severely dysfunctional behavior [12,13]. Early pregnancies and re-victimization occur at higher odds than in the general population $[14,15]$.

Given these negative consequences, it is obvious that abuse-related PTSD should be treated at an early stage. For children, there already exist evaluated interventions: a meta-analysis of 39 studies analyzing treatment results for children and adolescents with psychiatric symptoms after CSA reported a general effect size of $g=0.77$ for PTSD symptoms in the 5 studies with controlled designs and an untreated comparison control group. For the other studies, the authors report pre-post effect sizes of $g=1.13$ for PTSD [16]. In contrast, few randomized controlled trials (RCTs) for PTSD therapy of adolescents were included in this meta-analysis - only two studies enrolling adolescents over 14 years of age used control conditions (waiting list/ supportive treatment) and randomization $[17,18]$. However, as the mean age in both studies was around 11 years, the majority of participants were children. To our knowledge, there exist only five studies examining treatment effects on post-traumatic stress symptoms after CPA or CSA among adolescent patients exclusively [19-23]. Being the first of their kind, these studies are undoubtedly important. However, most of them have methodological deficits like caseness not defined by PTSD diagnoses or the use of insufficient diagnostic instruments. Only the study of Foa et al. [23] meets the standards for controlled studies, although it only focusses on girls. In summary, adolescents beyond the age of 14 are inadequately represented in PTSD research so far.

Thus, the major goal of the presented project herein is to provide information on treatment efficacy in this understudied group. A highly effective cognitive behavioral therapy manual for adults, Cognitive Processing Therapy (CPT [24]), was adapted for adolescent patients with CSA/CPArelated PTSD [25] - the Developmentally Adapted Cognitive Processing Therapy (D-CPT). Four major adjustments were integrated in the protocol. First of all, the treatment intensity was increased by administering the middle part of the protocol in high frequency (approximately 15 sessions during four weeks) to enhance the adolescents' motivation; applying treatment in such intensity was reported to be superior to the usual scheme of one session per week [26]. Second, a commitment phase was included in the protocol in order to further build treatment motivation and to enhance therapeutic alliance as well as to establish the formal framework that is needed to conduct therapy. As survivors of childhood abuse often experience difficulties in emotion regulation, behavior and emotion management techniques, as used within Dialectical Behavior Therapy for PTSD (DBT-PTSD $[27,28]$ ), were also integrated in D-CPT. Finally, special consideration was given to developmental tasks affecting the patient's entire life, such as career choice, vocational training, and romantic relationships. Adolescent patients are at high risk of school or secondary education drop out, of starting relationships with abusive partners, or of becoming re-victimized. Therefore, we decided to specifically address these issues at the end of treatment.

D-CPT has already been successfully piloted; nearly all of the 12 participating patients showed large reductions of posttraumatic stress symptoms and, moreover, comorbid symptoms improved significantly as well [25]. In October 2012, our research group started its work on the RCT and the associated studies. This article introduces its study protocol.

\section{The current trial}

The overall aim of this RCT is to demonstrate the efficacy of a newly adapted psychotherapy intervention protocol in comparison to treatment as usual (TAU) in the understudied population of physically and sexually abused adolescents and young adults suffering from PTSD. Severity of PTSD symptoms before and after therapy serves as a primary outcome. Secondary goals are to evaluate changes in general psychopathology and comorbidity, such as depression, borderline personality features, and dissociation. Thirdly, the trial will allow for an estimation of the efficacy of TAU in Germany, on which currently no data is available.

Adjunct projects will provide new insights in questions beyond treatment efficacy; electroencephalographic (EEG) parameters are collected in an experiment which aims to find neuronal correlates of threat processing evoked by different categories of emotional words. Preliminary studies could show that anxiety disorders modify the way threatening information is processed [29]. By adapting these studies to the current project, multiple research questions may be answered. Namely, it will be possible to investigate differences in information processing between healthy subjects (control group), traumatized but healthy subjects (trauma control group), and traumatized subjects with PTSD (experimental group). In addition to this, the influence of $\mathrm{D}-\mathrm{CPT}$ on information processing can be assessed on a psychophysiological level. 
On the basis of questionnaires and videotaped sessions, potential predictors of treatment success are analyzed. As there is little research about handling PTSD in adolescents, also important variables influencing therapy outcome, such as treatment adherence, therapist competence, and working alliance, are not well studied [30]. Equally sparse is data about societal costs for the treatment of PTSD and/or mental comorbidities like depression or anxiety disorders (direct costs) as well as PTSD-related loss of productivity (indirect costs). No study has ever investigated the indirect costs resulting from PTSD in adolescents and, so far, no study has analyzed any cost data of PTSD or PTSD-treatment in Germany. Using different questionnaires, these issues are addressed in the D-CPT-project.

Concerning the genetic basis of PTSD and therapy outcome, there are only very few (though promising) clinical studies indicating epigenetic changes in PTSD in humans (for example [31]). In our study, the analysis of epigenetic profiles of PTSD patients by saliva samples during the course of therapy is realized via high-throughput epigenetic profiling.

\section{Methods/design \\ Trial design}

D-CPT is an open, rater-blinded, multicenter, 2-arms RCT comparing D-CPT to TAU in Germany. There are five assessments: at baseline, within the treatment (approx. 8 weeks after beginning), directly after end of treatment (post-treatment) as well as 3 and 6 months after end of treatment (follow-up 1 and 2).

\section{Study centers}

The D-CPT project is designed as a multicenter study with Rita Rosner (University Eichstätt-Ingolstadt) as principal investigator. Treatment is offered in three university outpatient clinics: Freie Universität Berlin (supervised by Babette Renneberg), Goethe University Frankfurt am Main (supervised by Regina Steil), and University Eichstätt-Ingolstadt (supervised by Rita Rosner). Moreover, there are four partner projects at different research sites. Besides being a clinical center, the work group at Goethe University Frankfurt am Main (Regina Steil) studies potential predictors of treatment outcome. The analysis of epigenetic profiles of PTSD patients by saliva samples during the course of therapy is the task of a research group from the Max-Planck Institute of Psychiatry in Munich (Ulrike Schmidt). At the University Medical Center Hamburg-Eppendorf (Hans-Helmut König), direct and indirect costs of the disorder are evaluated. Bielefeld University (Frank Neuner) examines the neurophysiological processing of threat cues in PTSD and their respective changes in the course of the treatments. The DCPT network and its study centers are displayed in Figure 1.

\section{Participants}

Adolescents and young adults aged 14 to 21 years may take part in the study. For inclusion, PTSD as a primary diagnosis after experiencing CSA and/or CPA beyond the age of three, according to the definition of the American Psychological Association [32], is required. To be diagnosed with PTSD, patients must meet $1 \mathrm{~B}$-criterion, $2 \mathrm{C}$-criteria, and 2 D-criteria in the Clinician Administered PTSD Scale for Children and Adolescents (CAPS-CA [33]). A stable psychopharmacological medication is necessary at study entry, i.e., in the last 3 weeks there was either no or a constant psychopharmacological medication. Moreover, living in safe conditions, a sufficient knowledge of German language, as well as an informed consent from the parents/legal guardians (if under 18) and the participant is compulsory.

Exclusion criteria are:

- Acute suicidality within the last 6 months

- Life threatening self-harming behavior within the last 6 months

- Substance-related or organic mental disorder

- Pervasive developmental disorder

- Acute or lifetime diagnosis of a psychotic disorder according to the Diagnostic and Statistical Manual of Mental Disorders, Fourth Edition (DSM-IV-TR [34])

- Acute or lifetime diagnosis of a bipolar disorder according to DSM-IV-TR

- Current diagnosis of substance dependence according to DSM-IV-TR (abstinence $<6$ months)

- Mental retardation (IQ $\leq 75)$

- Simultaneous psychological or psychiatric treatment

\section{Planned interventions}

Patients are enrolled either to TAU or D-CPT. Patients in TAU will be advised to look for treatment with other therapists in the respective areas. A detailed instruction to find a psychotherapist via internet will be given to the client by the coordinator of the respective study center. TAU patients will be monitored at the corresponding measurement points planned for D-CPT. After follow-up 1, patients can be enrolled into D-CPT if they wish to do so.

D-CPT is organized into four phases [25]. The treatment begins with a commitment phase comprising 5 sessions in 4 weeks. The aim of this phase is to build a therapeutic alliance and to increase and test the adolescents' motivation for treatment. Safety plans are developed and anti-suicide contracts are signed. In phase 2, patients learn to tolerate and control intensive trauma-related emotions without acting in a dysfunctional way. For this reason, an emotion regulation training based on DBTPTSD [27] is applied with 6 sessions in 4 weeks. Phase 3 comprises 15 sessions of CPT, according to Resick et al. [24], administered in high frequency (within 4 weeks); this is the central part of D-CPT. Dysfunctional beliefs related 


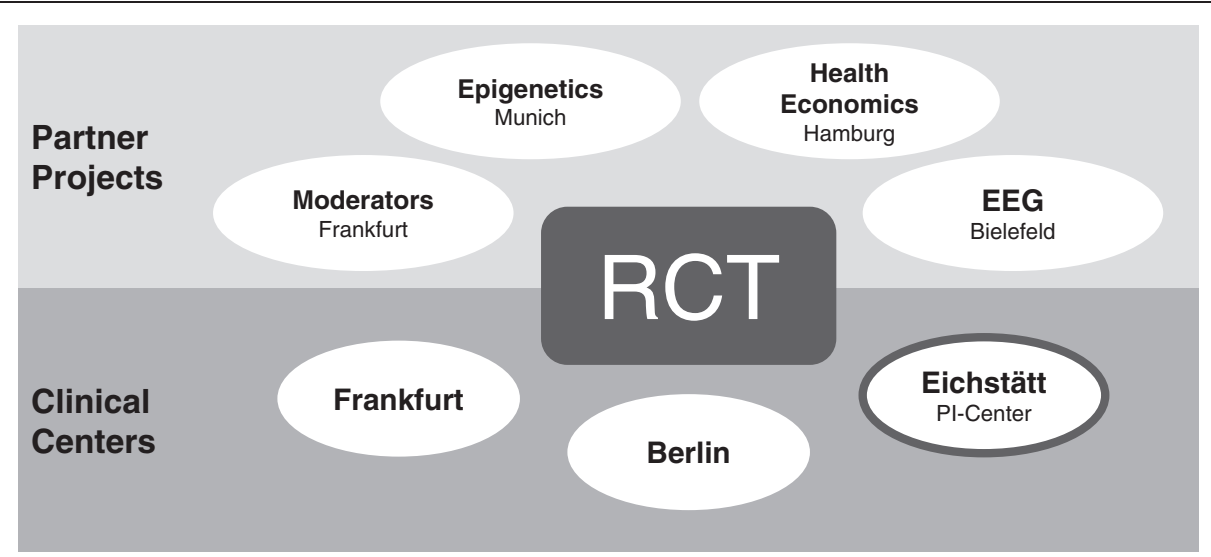

Figure 1 The D-CPT network and its study centers. RCT, Randomized controlled trial; PI, Principal investigator.

to the traumatic experiences are identified and modified by cognitive restructuring. In the last phase of treatment, developmental tasks specific to adolescence are introduced in 4 sessions during 4 weeks (one weekly session). The goal of this final therapy phase is to minimize the risk of future victimization and/or to prevent the choice of abusive partners. Moreover, the last treatment sessions focus on supporting the patient to complete education and to get an adequate vocational training. In sum, D-CPT comprises 30 sessions with 6 optional sessions for crisis intervention or communication with care givers or other institutions. Table 1 summarizes the treatment design and content. Adherence to D-CPT will be ensured by manualization, continuous local as well as central supervision, and ratings of therapeutic adherence and competence of random samples of videotaped sessions. Therapists are trained within one 4-day and one 2-day workshop. After attending the first workshop, every therapist treats at least one pilot case.

\section{Outcome measurements \\ Primary outcome}

Severity of PTSD is measured at each assessment point using the CAPS-CA [33] (in German [35]). The CAPS$\mathrm{CA}$ is a structured clinical interview which rates frequency and intensity of PTSD symptoms on a scale ranging from 0 (never) to 4 (daily or almost daily) and from 0 (none) to 4 (extreme), respectively. According to the German interview guidelines [35], a symptom is recorded as present if both frequency and intensity are at least rated with 1 .

Table 1 Overview of D-CPT

\begin{tabular}{|c|c|c|c|}
\hline Week & Sessions & Phase & Details \\
\hline \multirow[t]{3}{*}{$1-4$} & 5 & Commitment & Therapy contract, emergency plan \\
\hline & & & Lifeline \\
\hline & & & Therapy goals \\
\hline \multirow[t]{3}{*}{$5-8$} & 6 & Emotion-regulation & Monitoring and identifying dysfunctional behavior and its long-term consequences \\
\hline & & & Using distress tolerance skills \\
\hline & & & Education about emotions \\
\hline \multirow[t]{5}{*}{$9-12$} & 15 & Intensive CPT & PTSD symptoms and psychological processes \\
\hline & & & Identifying maladaptive beliefs ("stuck points") \\
\hline & & & Remembering the traumatic event through written accounts \\
\hline & & & Challenging stuck points \\
\hline & & & Focusing on special themes including safety, trust, control, esteem, and intimacy \\
\hline \multirow[t]{4}{*}{$13-16$} & 4 & Developmental tasks & Education about potentially abusive partners \\
\hline & & & Education-focused help \\
\hline & & & Inclusion of social network \\
\hline & & & Review \\
\hline
\end{tabular}




\section{Secondary outcome}

A self-rating of PTSD symptoms is obtained weekly within therapy and at every assessment point using the University of California Los Angeles PTSD Reaction Index (UCLA [36], in German [37]).

At pre- and post-treatment as well as at follow-up 1 and 2 assessments, psychiatric comorbidity is measured with the Structured Clinical Interview for DSM Disorders (SCID $[38,39]$, in German [40]), further important parts of the Diagnostic Interview for Mental Disorders in Childhood and Adolescence (Kinder-DIPS [41]), and the nicotine section of the Expert System for Diagnosing Mental Disorders (DIA-X [42]).

Moreover, patients are asked to complete a wide range of questionnaires measuring comorbidity and related problems at every assessment before and after treatment. Among these are:

- Trauma Symptom Checklist for Children (TSCC [43], in German [44])

- Beck Depression Inventory (BDI-II [45], in German [46])

- Adolescent Dissociative Experiences Scale (A-DES [47], in German [48])

- Borderline Symptom List 23 (BSL-23 [49])

- Youth Self-Report (YSR [50], in German [51])

- Modified version of the Client Sociodemographic and Service Receipt Inventory (CSSRI [52], in German [53])

- EuroQol 5 Dimensions (EQ-5D [54], in German [55])

Besides, saliva samples are collected for high-throughput epigenetic profiling and psychophysiological parameters are assessed by EEG - each at baseline and follow-up 1 .

\section{Additional measures}

Participants are tested with the Culture-Fair Intelligence Test (CFT-20-R [56]), which ensures that patients meet the cognitive requirements for treatment.

Detailed information about abuse are obtained by the Childhood Trauma Questionnaire (CTQ [57], in German [58]) and the Interview for Traumatic Events in Childhood (ITEC [59]). The ITEC is a retrospective, semi-structured interview for childhood maltreatment that provides dimensional scores for the severity of sexual, physical, and emotional abuse as well as emotional and physical neglect.

For the examination of mediators and moderators, like therapeutic alliance, maladaptive beliefs, and mental images, the Helping Alliance Questionnaire [60] (in German [61]), the Post-traumatic Maladaptive Beliefs Scale for Youth (PMBSY; Matulis S, Resick PA, Steil R, personal communication, 2012), and an instrument for the measurement of mental images (FVB; Schreiber F, Gutermann J, Matulis S, Steil R, personal communication, 2012) are used. Emotion regulation is assessed using the Affective Style Questionnaire (ASQ [62], in German [63]) and attachment is studied by the Experiences in Close Relationships - Revised Questionnaire (ECR-R [64], in German [65]). Therapeutic adherence and competence are rated on the basis of randomly drawn videographies of treatment sessions by independent raters using two newly developed rating scales, the D-CPT Adherence Scale [66] and the Cognitive Therapy Competence Scale for D-CPT [66].

\section{Procedure}

Patients will be sent by private practitioners, hospitals, school psychologists, and children' services as well as child and adolescent mental health services. Homepages, newspaper reports, and leaflets in schools will support individual referrals. In a first interview, patients are screened for inclusion and exclusion criteria. If suitable, patients are informed about study conditions and asked to give informed consent. When this requirement is met, pre-diagnostic procedures comprising SCID, Kinder-DIPS, DIA-X, CAPS-CA, ITEC, CFT-20-R, several questionnaires (YSR, CSSRI, EQ-5D, ECR-R, ASQ, BDI-II, A-DES, BSL-23, CTQ, UCLA, TSCC, PMBSY, FVB), saliva sampling, and an EEG session are carried out. Patients are randomized to either TAU or D-CPT subsequently. After emotion-regulation training, patients in $\mathrm{D}-\mathrm{CPT}$ are invited to complete process measures including the CAPS-CA and UCLA. For patients in TAU, this measurement is arranged around 8 weeks after randomization. After the end of treatment, post-diagnosis (directly after treatment), follow-up 1 (3 months after end of treatment), and follow-up 2 (6 months after end of treatment) measurements take place. These involve the SCID, KinderDIPS, DIA-X, CAPS-CA, and several questionnaires (YSR, CSSRI, EQ-5D, ECR-R, ASQ, BDI-II, A-DES, BSL-23, UCLA, TSCC, PMBSY, FVB) at each assessment point, as well as saliva collection and EEG at follow-up 1. These assessments are conducted by independent and trained interviewers who are blind to the treatment condition. Figure 2 shows the flow diagram of participant recruitment according to CONSORT [67].

\section{Sample size calculation}

A type I error of 0.05 and a statistical power of 0.8 were used for sample size calculations. As there exists no effect size estimation for TAU in Germany, we suggest an effect size of $d=0.3$. Based on the data reported by Harvey and Taylor [16], an effect size of $d=0.9$ was assumed for DCPT. Thus, two groups of $n=45$ patients are required for the trial. No adjustment for drop-out is necessary as last observation carried forward substitution of the major study endpoint will be performed during the intention-totreat analysis. 


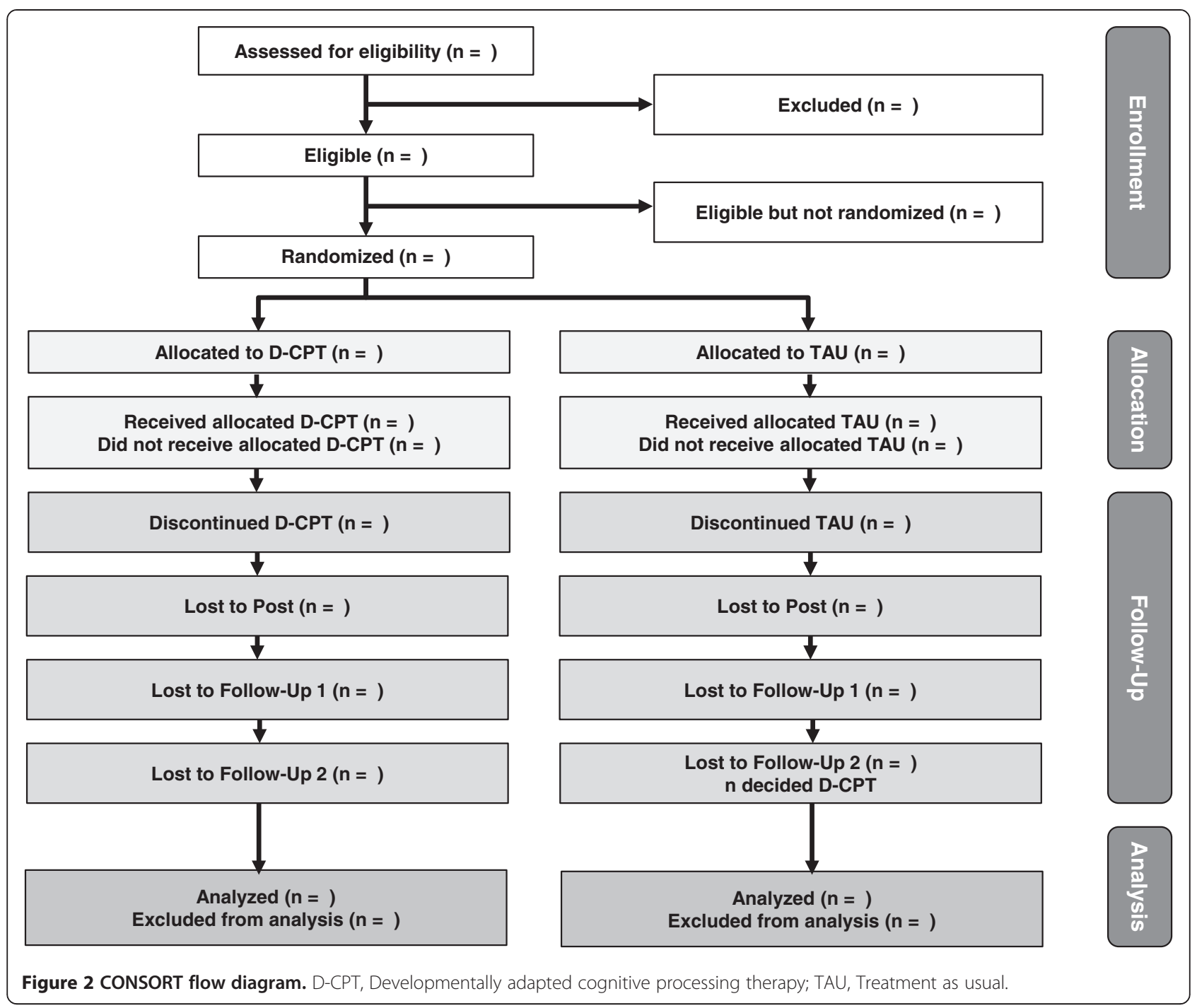

\section{Statistical analysis}

To test the hypothesis that D-CPT is superior to TAU, a confirmatory analysis of the study will be calculated as a $t$ test of post-treatment scores of the CAPS-CA. The critical value of alpha will be set to 0.05 . Additional analyses focusing on secondary study endpoints or on generating hypotheses for subsequent studies will be performed on an exploratory basis using up-to-date sophisticated statistical models.

\section{Safety and ethical issues}

The trial has been approved by the Ethics Committee of Catholic University Eichstätt-Ingolstadt and is conducted according to the ICH Guideline for Good Clinical Practice (ICH-GCP [68]). Prior to assessments, all patients are informed about study design and procedures including randomization, probable side effects, and the possibility of ending the participation at any time without disadvantages. Informed consent has to be provided by participating patients and by their parents or guardian if the adolescent is younger than 18 years of age. For safety reasons, suicidal tendencies are constantly observed during assessment and treatment. If necessary, patients will be referred to cooperating clinics. Serious adverse events (SAEs) are continuously monitored and reported through the whole trial. In correspondence with the ICH-GCP, the following incidents are defined as SAEs:

- A physical disease with the risk of permanent impairment and a following medical intervention

- Inpatient somatic hospitalization with a minimum of one overnight stay or prolongation of existing hospitalization

- Inpatient psychiatric hospitalization with a minimum of one overnight stay or prolongation of existing hospitalization (including emergency accommodation/crisis intervention service) 
- Intoxication with psychotropic substances (including alcohol) and following medical intervention

- Delinquency with judiciary or police contact

- External aggression to property (statutory offense)

- External aggression to persons (statutory offense)

- Intentional self-harming behavior requiring medical aid

- Attempted suicide

- Re-victimization including all traumatic events according to DSM-IV-TR

In case of a SAE, the responsible therapist or study coordinator reports the event to his/her supervisor and an independent Data and Safety Monitoring Board (DSMB). The decision, whether there is a causal link between treatment and occurrence of SAEs, is taken by the DSMB. If severe safety concerns become apparent to the DSMB, the trial will be stopped.

If an investigator has ethical concerns because of the performance at one of the centers, the coordinating investigator or the co-principal investigator will be informed immediately. The coordinating investigator and the coprincipal investigator are authorized to discontinue interventions in a trial center because of insufficient and/or inadequate recruitment, insufficient quality of data, or specific problems unforeseeable in advance which render the continuation of the study impossible at that specific center.

\section{Discussion}

Although the severe psychopathological consequences of sexual and/or physical abuse are known, only few studies report on the results of trauma focused psychotherapy for adolescents [19-23]. Consequently, chances were hitherto missed to administer effective treatments in early life to prevent the development of severe comorbidity or re-victimization. Reasons for the lack of studies are manifold, and might be due to difficulties in psychological assessments differing for adolescents and young adults, as well as to legal issues around different licensing procedures for therapists treating children and adolescents and those treating adult patients. Moreover, the lack of studies might be related to the characteristics of adolescents, whose motivational instability, rapid and intensive mood changes, and disentanglement from family structures have the potential to impede treatment participation and completion. The only published study on a similar methodological level needed 6 years of randomization to finally include 61 adolescent girls [23]. This highlights once more the specific problems in this age group. To bridge this knowledge gap by introducing a developmentally adapted CPT is the goal of the D-CPT network. The novelty of D$\mathrm{CPT}$ is the adaption of the CPT procedure to the group of traumatized adolescents by increasing treatment intensity, integrating emotion-regulation-training, and finally by considering developmental tasks. The overall aim of the project is to evaluate this new therapy. Besides, the network aims to address several other heavily underexplored issues like variables influencing treatment outcome, epigenetics, health economics, and neurophysiological processing.

Taken together, the innovative character of our project is represented in i) a study sample of adolescents and young adults suffering from PTSD, ii) the developmentally adapted version of CPT (D-CPT) whose feasibility for youth within the German health system will be evaluated, and iii) measurement of the so far unknown effectiveness of TAU in Germany, which will be estimated.

Moreover, issues addressed in the D-CPT partner projects have a pioneering value. Predictors of treatment success like treatment adherence, therapist competence, and working alliance are not as well studied in PTSD as in other disorder specific interventions [30]. The same applies to epigenetics and PTSD; to our knowledge, the D-CPT study will be the first clinical study analyzing epigenetic profiles of PTSD patients during the course of therapy [69] and it will be the first survey screening for epigenetic markers for PTSD in adolescents and young adults. Likewise, research on health economic evidence is rare for PSTD. Last but not least, the novelty in the examination of neurophysiological processing of threat cues in PTSD lies in the differentiation between maltreated subjects with and without psychopathology.

In summary, the D-CPT project will provide insight in a wide range of different issues concerning PTSD and its treatment in adolescents and young adults. We are confident that, in the long term, it will contribute to the improvement of psychotherapeutic care for this therapeutically underserved population.

The project is part of a comprehensive network about behavioral disorders related to violence, neglect, maltreatment, and abuse in childhood and adolescence.

\section{Trial status}

The trial started in October 2012 and will end in 2015/ 2016. Patient enrolment is ongoing with 31 of the 90 participants having been recruited.

\footnotetext{
Abbreviations

BDI-II: Beck Depression Inventory; ASQ: Affective Style Questionnaire;

CAPS-CA: Clinician Administered PTSD Scale for Children and Adolescents;

CPA: Childhood physical abuse; CPT: Cognitive Processing Therapy;

CSA: Childhood sexual abuse; CSSRI: Client Sociodemographic and Service Receipt Inventory; DBT: Dialectical Behavior Therapy; D-CPT: Developmentally Adapted Cognitive Processing Therapy; DIA-X: Expert System for Diagnosing Mental Disorders; DSMB: Data and Safety Monitoring Board; DSM-IV-TR: Diagnostic and Statistical Manual of Mental Disorders, Fourth Edition; ECR-R: Experiences in Close Relationships - Revised; EEG: Electroencephalogram; EQ-5D: EuroQol 5 Dimensions; FVB: Fragebogen zur Erfassung mentaler Vorstellungsbilder; ICH-GCP: ICH Guideline for Good Clinical Practice; Kinder-DIPS: Diagnostic Interview for Assessment of Psychological Disorders in Childhood and Adolescence; PI: Principal Investigator; PMBSY: Post-traumatic Maladaptive Beliefs Scale for Youth; PTSD: Post-traumatic stress disorder;

RCT: Randomized controlled trial; SAE: Serious adverse event;
} 
SCID: Structured Clinical Interview for DSM Disorders; TAU: Treatment as usual; UCLA: University of California Los Angeles PTSD Reaction Index; YSR: Youth Self-Report.

\section{Competing interests}

The authors declare that they have no competing interests.

\section{Authors' contributions}

$\mathrm{RR}$ is the Principal Investigator on the D-CPT trial. RR and RS did the major work on the design of the RCT and oversee data collection at their clinical center. HHK, FN, US, and RS are Project Coordinators on the trial and participated in the design of the study. All authors read and approved the final manuscript.

\section{Acknowledgements}

The study is funded by the German Federal Ministry of Education and Research (BMBF; 01KR1204A). The authors thank Babette Renneberg from Freie Universität Berlin for the supervision of one of the three university outpatient clinics and the support to bring the study to a successful end. We thank Eline Rimane for transferring the proposal and trial registration into the first draft of the manuscript.

\section{Author details}

'Department of Clinical and Biological Psychology, Catholic University Eichstätt-Ingolstadt, Ostenstr. 25, Eichstätt 85071, Germany. ${ }^{2}$ Department of Health Economics and Health Services Research, University Medical Center Hamburg-Eppendorf, Martinistr. 52, Hamburg 20246, Germany. ${ }^{3}$ Department of Clinical Psychology and Psychotherapy, Bielefeld University, Bielefeld 33501, Germany. ${ }^{4}$ Max-Planck-Institute of Psychiatry, Kraepelinstr. 2-10, Munich 80804, Germany. ${ }^{5}$ Department of Clinical Psychology and Psychotherapy, Institute of Psychology, Goethe University Frankfurt, Varrentrappstr. 40-42, Frankfurt am Main 60054, Germany.

Received: 7 February 2014 Accepted: 6 May 2014

Published: 29 May 2014

\section{References}

1. Pereda N, Guilera G, Forns M, Gomez-Benito J: The prevalence of child sexual abuse in community and student samples: a meta-analysis. Clin Psychol Rev 2009, 29:328-338.

2. Annerback EM, Sahlqvist L, Svedin CG, Wingren G, Gustafsson PA: Child physical abuse and concurrence of other types of child abuse in Sweden-Associations with health and risk behaviors. Child Abuse Negl 2012, 36:585-595.

3. Elklit A: Victimization and PTSD in a Danish national youth probability sample. J Am Acad Child Adolesc Psychiatry 2002, 41:174-181.

4. Finkelhor D, Turner H, Ormrod R, Hamby SL: Violence, abuse, and crime exposure in a national sample of children and youth. Pediatrics 2009, 124:1411-1423.

5. Hawkins AO, Danielson CK, de Arellano MA, Hanson RF, Ruggiero KJ, Smith DW, Saunders BE, Kilpatrick DG: Ethnic/racial differences in the prevalence of injurious spanking and other child physical abuse in a national survey of adolescents. Child Maltreat 2010, 15:242-249.

6. Stirling J Jr, Amaya-Jackson L: Understanding the behavioral and emotional consequences of child abuse. Pediatrics 2008, 122:667-673.

7. Fergusson DM, Boden JM, Horwood LJ: Exposure to childhood sexual and physical abuse and adjustment in early adulthood. Child Abuse Negl 2008, 32:607-619.

8. Gilbert R, Widom CS, Browne K, Fergusson D, Webb E, Janson S: Child maltreatment 1. Burden and consequences of child maltreatment in high-income countries. Lancet 2009, 373:68-81.

9. Cutajar MC, Mullen PE, Ogloff JR, Thomas SD, Wells DL, Spataro J: Psychopathology in a large cohort of sexually abused children followed up to 43 years. Child Abuse Negl 2010, 34:813-822.

10. McLeer SV, Deblinger E, Henry D, Orvaschel H: Sexually abused children at high risk for post-traumatic stress disorder. J Am Acad Child Adolesc Psychiatry 1992, 31:875-879.

11. McLeer SV, Dixon JF, Henry D, Ruggiero K, Escovitz K, Niedda T, Scholle R: Psychopathology in non-clinically referred sexually abused children. J Am Acad Child Adolesc Psychiatry 1998, 37:1326-1333.

12. Giaconia RM, Reinherz HZ, Silverman AB, Pakiz B, Frost AK, Cohen E: Traumas and posttraumatic stress disorder in a community population of older adolescents. J Am Acad Child Adolesc Psychiatry 1995, 34:1369-1380.

13. Essau CA, Conradt J, Petermann F: Häufigkeit der Posttraumatischen Belastungsstörung bei Jugendlichen: Ergebnisse der Bremer Jugendstudie. Z Kinder Jugendpsychiatr Psychother 1999, 27:37-45.

14. Barnes JE, Noll JG, Putnam FW, Trickett PK: Sexual and physical revictimization among victims of severe childhood sexual abuse. Child Abuse Negl 2009, 33:412-420.

15. Noll JG, Shenk CE, Putnam KT: Childhood sexual abuse and adolescent pregnancy: a meta-analytic update. J Pediatr Psychol 2009, 34:366-378.

16. Harvey ST, Taylor JE: A meta-analysis of the effects of psychotherapy with sexually abused children and adolescents. Clin Psychol Rev 2010 30:517-535.

17. King NJ, Tonge BJ, Mullen P, Myerson N, Heyne D, Rollings S, Martin R, Ollendick TH: Treating sexually abused children with posttraumatic stress symptoms: a randomized clinical trial. J Am Acad Child Adolesc Psychiatry 2000, 39:1347-1355.

18. Cohen JA, Mannarino AP, Knudsen K: Treating sexually abused children: 1 year follow-up of a randomized controlled trial. Child Abuse Negl 2005, 29:135-145.

19. Sinclair JJ, Larzelere RE, Paine M, Jones P, Graham K, Jones M: Outcome of group treatment for sexually abused adolescent females living in a group home setting: preliminary findings. J Interpers Violence 1995, 10:533-542.

20. Danielson CK, McCart MR, Walsh K, de Arellano MA, White D, Resnick HS Reducing substance use risk and mental health problems among sexually assaulted adolescents: A pilot randomized controlled trial. J Fam Psychol 2012, 26:628-635.

21. Tourigny M, Hébert M, Daigneault I, Simoneau AC: Efficacy of a group therapy for sexually abused adolescent girls. J Child Sex Abus 2005, 14:71-93.

22. Krakow B, Sandoval D, Schrader R, Keuhne B, McBride L, Yau CL, Tandberg $D$ : Treatment of chronic nightmares in adjudicated adolescent girls in a residential facility. J Adolesc Health 2001, 29:94-100.

23. Foa EB, McLean CP, Capaldi S, Rosenfield D: Prolonged exposure vs supportive counseling for sexual abuse-related PTSD in adolescent girls: a randomized clinical trial. JAMA 2013, 310:2650-2657.

24. Resick PA, Monson CM, Chard KM: Cognitive Processing Therapy: Veteran/ Military Version. Washington, DC: Department of Veterans' Affairs; 2008.

25. Matulis S, Resick PA, Rosner R, Steil R: Developmentally adapted cognitive processing therapy for adolescents suffering from posttraumatic stress disorder after childhood sexual or physical abuse: a pilot study. Clin Child Fam Psychol Rev 2014, 17(2):173-190.

26. Ehlers A, Clark DM, Hackmann A, Grey N, Liness S, Wild J, Manley J, Waddington L, McManus F: Intensive cognitive therapy for PTSD: a feasibility study. Behav Cogn Psychother 2010, 38:383-398.

27. Steil R, Dyer A, Priebe K, Kleindienst N, Bohus M: Dialectical behavior therapy for posttraumatic stress disorder related to childhood sexual abuse: a pilot study of an intensive residential treatment program. J Trauma Stress 2011, 24:102-106

28. Bohus M, Dyer AS, Priebe K, Krüger A, Kleindienst N, Schmahl C, Niedtfeld I, Steil R: Dialectical behaviour therapy for post-traumatic stress disorder after childhood sexual abuse in patients with and without borderline personality disorder: a randomised controlled trial. Psychother Psychosom 2013, 82:221-233.

29. Wabnitz P, Martens U, Neuner F: Cortical reactions to verbal abuse: eventrelated brain potentials reflecting the processing of socially threatening words. Neuroreport 2012, 23:774-779.

30. Barber JP, Triffleman E, Marmar C: Considerations in treatment integrity: Implications and recommendations for PTSD research. J Trauma Stress 2007, 20:793-805.

31. Uddin M, Aiello AE, Wildman DE, Koenen KC, Pawelec G, DeLos Santos R, Goldmann E, Galea S: Epigenetic and immune function profiles associated with posttraumatic stress disorder. Proc Natl Acad Sci U S A 2010, 107:9470-9475

32. American Psychological Association: Guidelines for psychological evaluations in child protection matters. Am Psychol 2013, 68:20-31.

33. Nader KO, Kriegler JA, Blake DD, Pynoos RS: Clinical Administered PTSD Scale, Child and Adolescent Version (CAPS-C). White River Junction, VT: National Center for PTSD; 1994.

34. APA (American Psychiatric Assosication): In Diagnostisches und Statistisches Manual Psychischer Störungen - Textrevision - DSM-IV-TR. Edited by Dt Bearb V, Sass H, Wittchen HU, Zaudig M, Houben I. Göttingen: Hogrefe; 2003. 
35. Steil R, Füchsel G: IBS-KJ. Interviews zu Belastungsstörungen bei Kindern und Jugendlichen. Diagnostik der akuten und der posttraumatischen Belastungsstörung. Göttingen: Hogrefe; 2006.

36. Steinberg AM, Brymer MJ, Decker KB, Pynoos RS: The University of California at Los Angeles post-traumatic stress disorder reaction index. Curr Psychiatry Rep 2004, 6:96-100.

37. Ruf M, Schauer M, Elbert T: UPID - UCLA PTSD Index for DSM IV. In Fragebögen zur Diagnostik psychischer Störungen des Kindes- und Jugendalters. Edited by Barkmann C, Schulte Markwort M, Brähler E. Göttingen: Hogrefe; 2010.

38. First MB, Spitzer R, Gibbon M, Williams J, Benjamin L: Structured Clinical Interview for DSM-IV Axis II Personality Disorders (SCID II). New York: Biometric Research Department; 1994.

39. First MB, Spitzer RL, Gibbon M, Williams JBW: Structured Clinical Interview for DSM-IV Axis I disorders (SCID I). New York: Biometric Research Department; 1997.

40. Wittchen $\mathrm{H}-\mathrm{U}$, Zaudig M, Frydrich T: Strukturiertes Klinisches Interview für DSM-IV. Achse I und II. Göttingen: Hogrefe; 1997.

41. Schneider S, Unnewehr S, Margraf J: Diagnostisches Interview bei psychischen Störungen im Kindes- und Jugendalter (Kinder-DIPS). 2nd edition. Berlin: Springer; 2009

42. Wittchen HU, Pfister H: DIA-X-Interviews: Manual für Screening Verfahren und Interview; Interviewheft Längsschnittuntersuchung (DIA-X-Lifetime); Ergänzungsheft (DIA-X-Lifetime); Interviewheft Querschnittsuntersuchung (DIA-X-12 Monate); Ergänzungsheft (DIA-X-12 Monate); PC-Programm zur Durchführung des Interviews (Längs- und Querschnittsuntersuchung); Auswertungsprogramm. Frankfurt: Swets \& Zeitlinger; 1997.

43. Briere J: Trauma Symptom Checklist for Children (TSCC) Professional Manual. Odessa, FL: Psychological Assessment Resources; 1996.

44. Matulis S, Loos L, Steil R: Validierung der deutschen adaptation der trauma symptom checklist for children [abstract]. Trauma \& Gewalt. Abstractband 2014, 146.

45. Beck AT, Steer RA, Brown GK: Manual for the Beck Depression Inventory-II. San Antonio, TX: The Psychological Corporation; 1996.

46. Hautzinger M, Keller F, Kühner C: BDI-II. Beck Depressions-Inventar Revision. Frankfurt: Harcourt Test Services; 2006.

47. Armstrong JG, Putnam FW, Carlson EB, Libero DZ, Smith SR: Development and validation of a measure of adolescent dissociation: the adolescent dissociative experiences scale. J Nerv Ment Dis 1997, 185:491-497.

48. Brunner R, Resch F, Parzer P, Koch E: Heidelberger Dissoziations-Inventar (HDI): Manual. Frankfurt/Main: Pearson; 2008

49. Bohus M, Kleindienst N, Limberger MF, Stieglitz R-D, Domsalla M, Chapman AL, Steil R, Philipsen A, Wolf M: The short version of the Borderline Symptom List (BSL-23): development and initial data on psychometric properties. Psychopathology 2009, 42:32-39.

50. Achenbach TM: Manual for the Youth Self-Report and 1991 Profile. Burlington: University of Vermont, Department of Psychiatry; 1991.

51. Döpfner M: Fragebogen für Jugendliche; deutsche Bearbeitung der Youth Self Report Form der Child Behavior Checklist (YSR). Köln: Arbeitsgruppe Kinder-, Jugend- und Familiendiagnostik; 1998

52. Chisholm D, Knapp MRJ, Knudsen HC, Amaddeo F, Gaite L, van Wijngaarden B: Client socio-demographic and service receipt inventory - European version: development of an instrument for international research: EPSILON Study 5. Br J Psychiatry Suppl 2000, 177:s28-s33.

53. Roick C, Kilian R, Matschinger H, Bernert S, Mory C, Angermeyer MC: German adaptation of the client sociodemographic and service receipt inventory - an instrument for the cost of mental health care. Psychiatr Prax 2001, 28:84-90.

54. Group EQ: EuroQol - a new facility for the measurement of healthrelated quality of life. Health policy 1990, 16(3):199-208.

55. von der Schulenburg JMG, Claes C, Greiner W, Uber A: Die deutsche Version des EuroQol-Fragebogens. Z Gesundh Wiss 1998, 6:3-20.

56. Weiß RH: Grundintelligenztest Skala 2 - Revision CFT 20-R. Göttingen: Hogrefe; 2006.

57. Bernstein DP, Fink L: Childhood Trauma Questionnaire. A retrospective selfreport. Orlando: The Psychological Corporation; 1998.

58. Bader K, Hänny C, Schäfer V, Neuckel A, Kuhl C: Childhood Trauma Questionnaire - Psychometrische Eigenschaften einer deutschsprachigen Version. Z Klin Psychol Psychother 2009, 38:223-230.

59. Lobbestael J, Arntz A, Harkema-Schouten P, Bernstein D: Development and psychometric evaluation of a new assessment method for childhood maltreatment experiences: the Interview for Traumatic Events in Childhood (ITEC). Child Abuse Negl 2009, 33:505-517.

60. Alexander LB, Luborsky L: The Penn Helping Alliance Scales. In The Psychotherapeutic Process: A Research Handbook. Guilford Clinical Psychology and Psychotherapy Series. Edited by Greenberg LS, Pinsof WM. New York, NY: Guilford Press; 1986:325-366.

61. Bassler M, Potratz B, Krauthauser H: Der "Helping Alliance Questionnaire" (HAQ) von Luborsky: Möglichkeiten zur Evaluation des therapeutischen Prozesses von stationärer Psychotherapie. Psychotherapeut 1995, 40:23-32.

62. Hofmann SG, Kashdan TB: The affective style questionnaire: development and psychometric properties. J Psychopathol Behav Assess 2010, 32:255-263.

63. Graser J, Bohn C, Kelava A, Schreiber F, Hofmann SG, Stangier U: Der "Affective Style Questionnaire" (ASQ): Deutsche Adaption und Validitäten. Diagnostica 2012, 58:100-111.

64. Fraley RC, Waller NG, Brennan KA: An item response theory analysis of selfreport measures of adult attachment. J Pers Soc Psychol 2000, 78:350-365.

65. Ehrenthal JC, Dinger U, Lamla A, Funken B, Schauenburg $\mathrm{H}$ : Evaluation der deutschsprachigen Version des Bindungsfragebogens "Experiences in Close Relationships - Revised" (ECR-RD). Psychother Psychosom Med Psychol 2009, 59:215-223.

66. Gutermann J, Schreiber F, Matulis S, Steil R: Reliabilität der Skalen zur Erfassung psychotherapeutischer Adhärenz und Kompetenz in der Behandlung von Jugendlichen mit Posttraumatischer Belastungsstörung (PTBS) nach Gewalterfahrungen [abstract]. Trauma \& Gewalt. Abstractband 2014, 145

67. Schulz KF, Altman DG, Moher D: CONSORT 2010 statement: updated guidelines for reporting parallel group randomized trials. Ann Intern Med 2010, 152:726-732.

68. ICH Harmonised Tripartite Guideline: Guideline for Good Clinical Practice E6 http://www.ich.org/fileadmin/Public_Web_Site/ICH_Products/Guidelines/ Efficacy/E6_R1/Step4/E6_R1_Guideline.pdf.

69. Schmidt U, Kaltwasser SF, Wotjak CT: Biomarkers in posttraumatic stress disorder: overview and implications for future research. Dis Markers 2013, 35:12.

doi:10.1186/1745-6215-15-195

Cite this article as: Rosner et al:: Developmentally adapted cognitive processing therapy for adolescents and young adults with PTSD symptoms after physical and sexual abuse: study protocol for a randomized controlled trial. Trials 2014 15:195.

\section{Submit your next manuscript to BioMed Central and take full advantage of:}

- Convenient online submission

- Thorough peer review

- No space constraints or color figure charges

- Immediate publication on acceptance

- Inclusion in PubMed, CAS, Scopus and Google Scholar

- Research which is freely available for redistribution 